\title{
The preventive role of transurethral antibiotic delivery in a rat model
}

\author{
This article was published in the following Dove Press journal: \\ Drug Design, Development and Therapy \\ 16 July 2012 \\ Number of times this article has been viewed
}

\author{
Hakki U Ozok' \\ Okan Ekim ${ }^{2}$ \\ Hakan Saltas ${ }^{3}$ \\ Ata T Arikok ${ }^{4}$ \\ Orkun Babacan ${ }^{5}$ \\ Levent Sagnak' \\ Hikmet Topaloglu' \\ Hamit Ersoy' \\ 'Department of Urology, ${ }^{3}$ Department \\ of Microbiology, ${ }^{4}$ Department of \\ Pathology, Diskapı Yildirim Beyazit \\ Training and Research Hospital, \\ Ministry of Health, Ankara, \\ Turkey; ${ }^{2}$ Department of Anatomy, \\ ${ }^{5}$ Department of Microbiology, Ankara \\ University Faculty of Veterinary \\ Medicine, Ankara, Turkey
}

Correspondence: Hakki Ugur Ozok

Kardelen Mah 2066 sok

Siyasal-2 Sit No 83, Batıkent

Yenimahalle, Ankara 06370, Turkey

Tel +905422424660

Fax +903I22 552086

Email drozok@gmail.com
Purpose: There is currently an emerging need for developing improved approaches for preventing urinary tract infections (UTIs) occurring during diagnostic or interventional procedures of the lower urinary tract. We aimed to establish a rat model to assess the use of transurethral antibiotic administration and to provide evidence that this could be used as a preventive therapy.

Methods: Animals received fosfomycin trometamol (FOF) either urethrally or orally prior to the procedure. A third group was generated as treatment controls and did not receive any medication. Urethral dilation was conducted to recapitulate an interventional procedure prior to intravesical Escherichia coli administration in all three groups. Finally, sham-operated animals were introduced as a fourth group which did not receive antibiotics or E. coli. Colony counts of urine and tissue cultures for the identification of E. coli and histopathological examinations of the bladder and prostate were conducted.

Results: Evaluation of infection intensities in cultures as well as histopathological examination of the bladder and prostate demonstrated a preventative role of transurethral FOF administration. In terms of efficiency, local administration of FOF was similar to oral administration.

Conclusions: These results suggest that transurethral antibiotic administration is a promising alternative for preventing UTIs occurring during diagnostic or interventional procedures of the lower urinary tract.

Keywords: cystitis, fosfomycin, infection, prostatitis, urinary tract

\section{Introduction}

Use of systemic antibiotics for preventing urinary tract infections occurring during or following diagnostic or minimally invasive interventions related to the urethra and bladder is only reasonable in the presence of risk factors. ${ }^{1,2}$ Symptomatic urinary tract infection (UTI) incidence is low following cystoscopy and/or during invasive surgical procedures conducted during cystoscopy and the level of evidence on antibiotics prophylaxis was reported to be low, if not moderate. ${ }^{3,4}$ On the other hand, the patient is considered to be at risk for further postcytoscopy bacteriuria or symptomatic UTI development in cases such as the presence of UTI prior to additional procedures, in cystoscopy leading to disruption of the mucosa (ie, internal urethrotomy or biopsy), or in the presence of certain comorbidities (eg, immune system-weakening conditions such as diabetes mellitus, chronic system disease, and use of immunosuppressive drugs). In these specific cases, there is indeed a systemic antibiotic prophylaxis requirement. ${ }^{1}$ Side effects and development of microbial resistance patterns are the major risk factors for the use of antibiotics. ${ }^{5}$ It is therefore crucial to consider alternative 
treatment strategies for the patients within the UTI risk group. Here, we hypothesized that transurethral administration of an anti-microbial agent prior to the diagnostic or minimal intervention of the bladder and urethra would be beneficial as it would act faster, lead to fewer side effects and therefore provide higher efficacy than systemic administration.

Fosfomycin trometamol (FOF) is an efficient antibiotic in uncomplicated UTIs with a single dose of oral application and is soluble in water. ${ }^{6}$ It can therefore be easily administered transurethrally due to these characteristics as an alternative to its conventional use. Furthermore, FOF was shown to be effective in Escherichia coli-induced UTIs with a single oral administration allowing us to test it in an animal model setting. ${ }^{7}$

In order to investigate whether local treatment of an antimicrobial agent could be beneficial in interventioninduced UTI in an animal model, here we established a rat model of transurethral delivery and tested, for the first time, the efficacy of transurethral administration of FOF.

\section{Materials and methods}

A total number of 42 male Wistar rats weighing approximately $300 \mathrm{~g}$ were used for this experiment. All rats had ad libitum access to food and water during a 12 hour light/dark cycle. All experimental procedures were approved by the Local Ethics Committee of Ankara University. Culture negative male Wistar rats were obtained from Ankara University Faculty of Medicine Experimental Animals Laboratory. FOF was directly supplied from the manufacturer (Drogsan, Istanbul, Turkey) in pure form and was freshly prepared in sterile saline. Dilation in the sham group was performed using the outer sheath of 18-gauge percutaneous entry needle (Matek, Ankara, Turkey). For other treatment purposes and E. coli administration the outer sheath of 26-gauge sterile polyurethane angiocath was used.

\section{Bacterial strains}

The uropathogenic E. coli J96 (O4:K6:H5) strain was used to generate the UTI and expressed, sfa, hly, fim, chf, tra, and usp genes.

\section{Preliminary study}

In order to establish the evaluation time and criteria for the cultures we conducted a preliminary time course study. Sensitivity of the E. coli J96 strain to FOF was evaluated by the Kirby-Bauer disc diffusion method based on Clinical and Laboratory Standards Institute criteria from inhibition zone diameters obtained on Mueller-Hinton agar (MH; Plasmatec,
Bristol, UK) in a laboratory environment. The obtained inhibition zone diameters were established as sensitive following the results of $\geq 16 \mathrm{~mm}$ for FOF based on Clinical and Laboratory Standards Institute criteria.

We then conducted a time course study to investigate the experimental cystitis and prostatitis developing process of the E. coli J96 strain. To this end, 18 culture negative male Wistar rats were divided into three groups and anesthetized with intramuscular ketamine $\mathrm{HCl}(80 \mathrm{mg} / \mathrm{kg})$ and xylazine $(10 \mathrm{mg} / \mathrm{kg})$. Next, the cannula was placed into the urethra and $0.3 \mathrm{~mL}$ of $E$. coli $\mathrm{J} 96$ strain with a dose of $10^{8}$ colonyforming units (CFU) was administered into the bladder of each rat. The agent was kept inside the bladder for 30 minutes by tying the penis with $2 / 0$ silk suture. Laparotomy was conducted on the animals at day 3 for the first group, day 7 for the second group, and day 14 for the third group with a vertical incision and bladder content was aspired with a 28-gauge needle aseptically. Bladder, prostate, and the abdominal part of the urethra were dissected for further analysis. E. coli infection and the effects of infection were investigated in urine sample cultures and tissue cultures as well as by using histopathological analysis of the bladder and prostate tissue.

\section{Main study}

Twenty-four rats were divided into four groups $(n=6$, rat/ group). All procedures were conducted under anesthesia as described above. FOF was administered to group 1 immediately before the procedure with the same method used in the preliminary study through a sterile catheter placed urethrally at $50 \mathrm{mg} / \mathrm{kg}$ concentration in $0.3 \mathrm{~mL}$ volume. Group 2 received $50 \mathrm{mg} / \mathrm{kg}$ oral FOF in $0.3 \mathrm{~mL}$ volume through an orogastric tube 3 hours prior to the procedure. Group 3 did not receive any medication and was used as a control group. Next, all three groups went through urethral dilation in order to recapitulate an interventional procedure. Then, FOF-sensitive E. coli J96 strain that had earlier been confirmed to develop cystoprostatitis in rats was administered with intravesical injection in $1 \times 10^{8} \mathrm{CFU} / \mathrm{mL}$ concentration to each of the first three groups. Furthermore, we generated a fourth group of sham animals that underwent a dilation procedure but did not receive any antibiotics or $E$. coli. In order to achieve $1 \times 10^{8} \mathrm{CFU} / \mathrm{mL}$ concentration of $E$. coli for the dilution procedure, the lyophilized strain was incubated on MacConkey agar for 24 hours at $37^{\circ} \mathrm{C}$. A single colony was picked and incubated in nutrient bouillon for 24 hours at $37^{\circ} \mathrm{C}$. Tenfold serial dilutions in sterile saline were performed. From each dilution, $0.1 \mathrm{~mL}$ of culture was spread on nutrient 
agar and incubated for another 24 hours at $37^{\circ} \mathrm{C} .{ }^{8}$ The correct dilution was determined by colony counts.

Subsequently, animals were left ad libitum for 3 days. Urine, bladder, and prostate samples were obtained at day 3 with the same method as the preliminary study. For the urine culture, samples were cultured in Levine's eosin-methylen blue agar (Levine's EMB; HiMedia, Mumbai, India). E. coli colonies were counted following 24 hours of incubation at $37^{\circ} \mathrm{C}$. Half of the collected bladder and prostate were used for histopathological examination; the other half was weighed, homogenized in $1 \mathrm{~mL}$ sterile saline for $1 \mathrm{mg}$ tissue, serially diluted, and cultured on EMB agars. In order to estimate the number of total bacteria in tissue (infection intensities of bladder and prostate), E. coli colonies in serial cultures were counted following 24 hours of incubation at $37^{\circ} \mathrm{C}$. In addition, polymerase chain reaction was conducted to confirm that the isolated strain was indeed the J96 strain by amplifying the virulent genes that J96 is believed to have. Bacterial DNA from urine samples was isolated using the GeneJET Genomic DNA purification kit (Fermentas, Burlington, ON) according to the manufacturers' instructions. ${ }^{9}$ Protocol and amplification conditions, which are described by Yamamoto et al, ${ }^{10}$ were modified and optimized from multiplex PCR to single PCR by Babacan $\mathrm{O}^{9}$, were used in this study. ${ }^{9-10}$ Primers targeting $s f a$, hly, cnf, traT, fim, and usp were generated according to earlier literature. ${ }^{9-12}$ Briefly, $10 \mu \mathrm{L}$ of amplified sample was loaded in $1.5 \%$ agarose gel (Prona, Madrid, Spain). Electrophoresis was conducted for 60 minutes at 180 volts., ${ }^{9,10}$

\section{Histopathological study}

The bladder and prostate tissue samples were immersion fixed in $10 \%$ formalin for 24 hours and embedded in paraffin. Paraffin blocks were sectioned at $4 \mu \mathrm{m}$ thickness and stained with hematoxylin and eosin. The stained specimens were then examined using Olympus BX51 microscope and scored by an observer blinded to the identity of the samples according to the classification presented in earlier literature. ${ }^{13,14}$ Briefly, sections were scored for bladder tissue as follows: grade 0, normal; grade 1, subepithelial cell inflammatory infiltration (focal and multifocal); grade 2 , edema and subepithelial cell inflammatory infiltration (diffuse); grade 3, marked subepithelial inflammatory cells with necrosis and neutrophils in and on bladder epithelium; grade 4, inflammatory cell infiltrate extends into muscle in addition to criteria for grade 3; and grade 5 , loss of surface epithelium (necrosis with full-thickness inflammatory cell infiltration). Also, prostate sections were scored as follows: grade 0 , normal; grade 1 , edema and infiltrates of polymorphonuclear (PMN) leukocytes in the prostate stroma; grade 2, PMN leukocytes in the prostate acini; grade 3, loss of epithelial and/or basement membrane architecture in the prostate acini; grade 4, hemorrhage; and grade 5 , tissue necrosis.

\section{Statistical methods}

Statistical significance for the group comparisons for bladder and prostate culture intensities were analyzed by using oneway ANOVA, followed by post-hoc comparisons using the Tukey test. The above-mentioned data were represented as the mean \pm the standard error of the mean. All other nominal and ordinary data were analyzed using the nonparametric Kruskal-Wallis test and groups were compared by a posthoc Dunn's multiple comparison test. Nominal data were expressed as median (min-max) for nominal variables and the ordinal data was represented as number of cases and/ or as percentage (\%). The $P$ value $<0.05$ was accepted as statistically significant.

\section{Results}

First, before testing the transurethral efficacy of FOF, in a preliminary experimental setup, we evaluated the sensitivity of the E. coli J96 strain against FOF and confirmed its effectiveness in developing cystitis and prostatitis in rats. Urine and tissue cultures as well as histopathological examination of the bladder and prostate tissue revealed cystitis and prostatitis in six animals already at day 3 (Table 1). We did not find significant exacerbation of the symptoms in any of the cultures or histology specimens,

Table I Comparison of urine cultures and tissue infection intensities between groups in preliminary study

\begin{tabular}{|c|c|c|c|c|c|c|c|c|}
\hline \multirow[t]{2}{*}{ Group } & \multicolumn{2}{|c|}{ Urine cultures } & \multicolumn{3}{|c|}{ Bladder infection intensities } & \multicolumn{3}{|c|}{ Prostate infection intensities } \\
\hline & $\mathbf{n}^{*}$ & $\mathbf{p}^{\prime}$ & $\mathbf{n}^{*}$ & CFU & $\mathbf{p}^{2}$ & $\mathbf{n}^{*}$ & CFU & $\mathbf{p}^{2}$ \\
\hline I & $6 / 6$ & & $6 / 6$ & $99,713( \pm 36,321)$ & & $6 / 6$ & $124,995( \pm 35,273)$ & \\
\hline 2 & $4 / 6$ & 0.455 & $3 / 6$ & $36,733( \pm 35,524)$ & 0.011 & $5 / 6$ & $54,616( \pm 41,289)$ & 0.014 \\
\hline 3 & $2 / 6$ & 0.061 & $2 / 6$ & $13,833( \pm 24,296)$ & 0.001 & $3 / 6$ & $30,666( \pm 35,000)$ & 0.001 \\
\hline
\end{tabular}

Abbreviations: $\mathrm{n}^{*}$, number of rats infected in each group; $\mathrm{P}^{1}$, Comparison of number of rats infected between group I and others by Fisher's exact test; $\mathrm{P}^{2}$, Comparison of CFU means between group I and others by one-way ANOVA analysis followed by Tukey post-hoc test; ANOVA, analysis of variance; CFU, colony-forming unit. 
but observed spontaneous resolution on days 7 and 14 and therefore conducted the treatment study by collecting data 3 days following the intervention. None of the animals died or were excluded from the experiment due to any procedure or treatment.

Next, we evaluated the bacterial growth and histopathological parameters among the transurethral administration, oral administration, control, and sham groups. First, we compared urine cultures as well as bladder and prostate tissue cultures in order to assess bacterial growth (Table 2). Urine cultures were positive in all animals (100\%) in the control group indicating that $E$. coli administration successfully led to UTI. Positive urine culture rates were $66.7 \%$, $33.3 \%$, and $0 \%$ in group 1 , group 2 , and the sham group, respectively, and reached significance $(P=0.004)$. We then analyzed the infection intensity in the bladder. All animals that received either transurethral or oral FOF administration demonstrated significantly lower intensity of bladder infection compared to the controls and were not statistically different from the sham group. Results from the analysis of prostate cultures were also in line with the findings from the bladder infection intensities. Each FOF administration route led to a significant decrease in infection as compared to controls. Similar to the bladder infection intensity results, although oral administration had a tendency to result in a higher recovery compared to transurethral administration, these differences between the prostate intensities following oral and transurethral FOF delivery were not statistically significant. Note that all cases were verified as the $E$. coli J96 strain by polymerase chain reaction suggesting that all findings were related to the experimental procedure, but did not occur spontaneously.

As a second outcome, we evaluated the tissue samples histopathologically. Infection stages in the bladder and prostate are demonstrated visually in Figures 1 and 2, respectively. As expected, all control animals represented a pathology related to the infection in both bladder and prostate tissue analysis. Except for two bladder tissue samples, the sham group demonstrated a normal histology. Both FOF administration groups led to either complete prevention of infection and were scored as 0 , or received a score ranging from $1-3$. The histopathological scores in the bladder revealed no significant difference between the delivery methods used for FOF administration $(P=0.071)$. In the bladder histopathology, while group 1, group 2, and the sham group were mostly staged as normal or focal/multifocal subepithelial inflammation (Figure 1A), a general picture was observed ranging from a complete layer of inflammatory cell infiltration to tissue necrosis in the control group (Figure 1C, D, and Table 3). However, all the groups had significantly lower histopathological scores than the control group (statistical differences between the control group and group 1, group 2, and the sham group were $P<0.001, P<0.001$, and $P<0.001$, respectively) (Table 3).

Analysis of the prostate tissue demonstrated that both transurethral and oral delivery of FOF led to a significant improvement in the pathology compared to treatment controls ( $P=0.007$ and $P=0.002$, respectively). Histopathological analyses of the prostate showed that the grades were mostly in the normal category in group 2 and the sham group (Figure 2A), and epithelium structure and/or basal membrane structure loss in prostate acini were observed in group 1 (Figure 2D). As expected, a severe picture was present leading to tissue necrosis in the control group (Figure 2E, F and Table 4). Oral FOF administration led to a significant improvement in the prostate pathology and this was not significantly different from the sham group $(P=0.140)$. On the other hand, this improvement was significantly better than the transurethral administration group $(P=0.007)$ and control group $(P=0.002)$. This suggests that oral FOF delivery leads to a better protection in the prostate tissue compared to transurethral delivery.

Table 2 Comparison of urine cultures and tissue infection intensities between groups in main study

\begin{tabular}{|c|c|c|c|c|c|c|c|c|}
\hline \multirow[t]{2}{*}{ Group } & \multicolumn{2}{|c|}{ Urine cultures } & \multicolumn{3}{|c|}{ Bladder infection intensities } & \multicolumn{3}{|c|}{ Prostate infection intensities } \\
\hline & n* & $\mathbf{p}^{\prime}$ & n* & CFU & $\mathbf{p}^{2}$ & n* & CFU & $\mathbf{p}^{2}$ \\
\hline 1 & $4 / 6$ & 0.455 & $3 / 6$ & $28,017( \pm 37,3 \mid 2)$ & $<0.001$ & $5 / 6$ & $56,283( \pm 39,852)$ & $<0.01$ \\
\hline 2 & $2 / 6$ & 0.061 & $2 / 6$ & $17,167( \pm 27,083)$ & $<0.001$ & $3 / 6$ & $32,333( \pm 35,998)$ & $<0.001$ \\
\hline Sham & $0 / 6$ & $<0.01$ & $1 / 6$ & $7483( \pm \mathrm{II}, 808)$ & $<0.001$ & $1 / 6$ & $5417( \pm 13,268)$ & $<0.001$ \\
\hline Control & $6 / 6$ & & $6 / 6$ & $104,7 \mid 3( \pm 32,537)$ & & $6 / 6$ & | 3,3328 $( \pm 4 \mid, 725)$ & \\
\hline
\end{tabular}

Abbreviations: $n^{*}$, number of rats infected in each group; $p^{\prime}$, Comparison of number of rats infected between control group and others by Fisher's exact test; $\mathrm{P}^{2}$, Comparison of CFU means between control group and others by variance analysis followed by Tukey post-hoc test; CFU, colony-forming unit. 


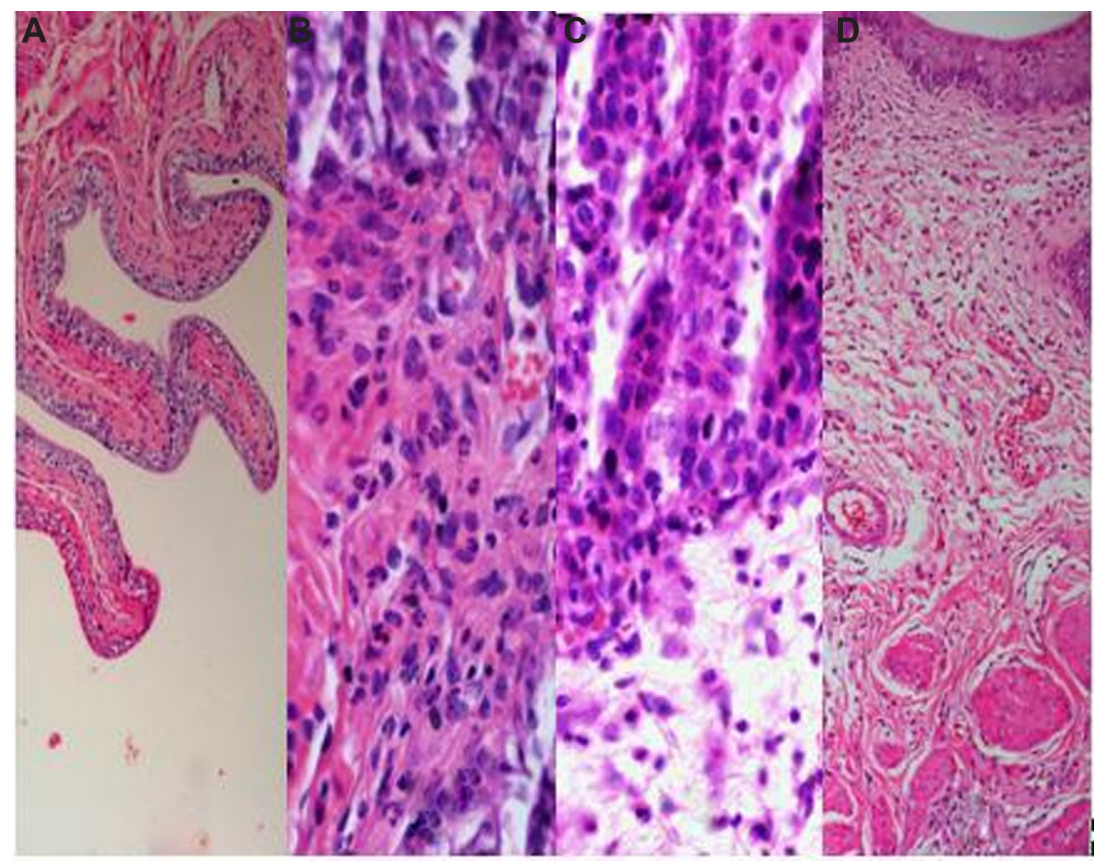

Figure I Representative images of different histopathological grades in the rat bladder tissue following UTI.

Notes: Each panel represents a different pathology scored according to the grading scale summarized in Table I. (A) Grade 0 inflammation (H\&E, $\times 40$ ); (B) Grade 2 inflammation (H\&E, $\times 40$ ); (C) Grade 3 inflammation (H\&E, $\times 10$ ); (D) Grade 4 inflammation (H\&E, $\times 10$ ).

Abbreviations: H\&E, hematoxylin and eosin; UTI, urinary tract infection.

\section{Discussion}

FOF is effective against the E. coli

\section{J96 strain in vitro and the appropriate} evaluation time is the third day

In an earlier study, in vitro FOF sensitivity of $E$. coli isolated from patients was found to be $97 \%$ in 236 clinical urinary E. coli specimens. ${ }^{15}$ In a more recent clinical study, among 1705 E. coli strains, $5 \%$ were found to be resistant to FOF. ${ }^{16}$ We therefore initially sought to confirm the sensitivity of the E. coli J96 strain and rule out the possibility of a resistance compromising the following experiments with FOF treatment.

The response of the host against UTI in animal models might lead to progressive spontaneous resolution. ${ }^{13}$ Hopkins et $\mathrm{al}^{13}$ demonstrated a significant spontaneous recovery against $E$. coli-mediated UTI in 6 out of 10 rat strains with different genetic backgrounds within 14 days. In our preliminary study we confirmed that indeed, the in-house bred Wistar rats exhibit spontaneous resolution 7 and 14 days postsurgery and therefore, in order to obtain an appropriate evaluation time in terms of both clinical and laboratory analysis we used the third day in the experimental cystitis and prostatitis model formed by the E. coli J96 strain. Spontaneous resolution observed on day 7 and 14 could have caused a conflict about whether the results were due to the antibiotics used or to spontaneous resolution.

\section{Transurethral delivery of FOF leads to decreased infection intensities and improved pathology}

When infection intensities were evaluated in urine and tissue cultures, we observed a significant prevention of infection intensities in the urine and tissue samples when FOF was introduced via the urethra. This protective effect was not significantly different from the sham group. Furthermore, we did not find any difference between oral and transurethral treatment paradigms in the urine and tissue infection rates. Oral administration led to a stronger protection in both urine and tissue cultures, although the results did reach statistical significance. Histopathological analysis of the bladder and prostate also confirmed the effectiveness of the transurethral administration when compared with the control group. Results of this analysis were in parallel to urine and tissue culture results when bladder and prostate histopathologies were assessed. This analysis led us to reveal detailed information about the form of the inflammation. In the light of these findings, we can conclude that transurethral administration of FOF provides significant prevention to the bladder and 


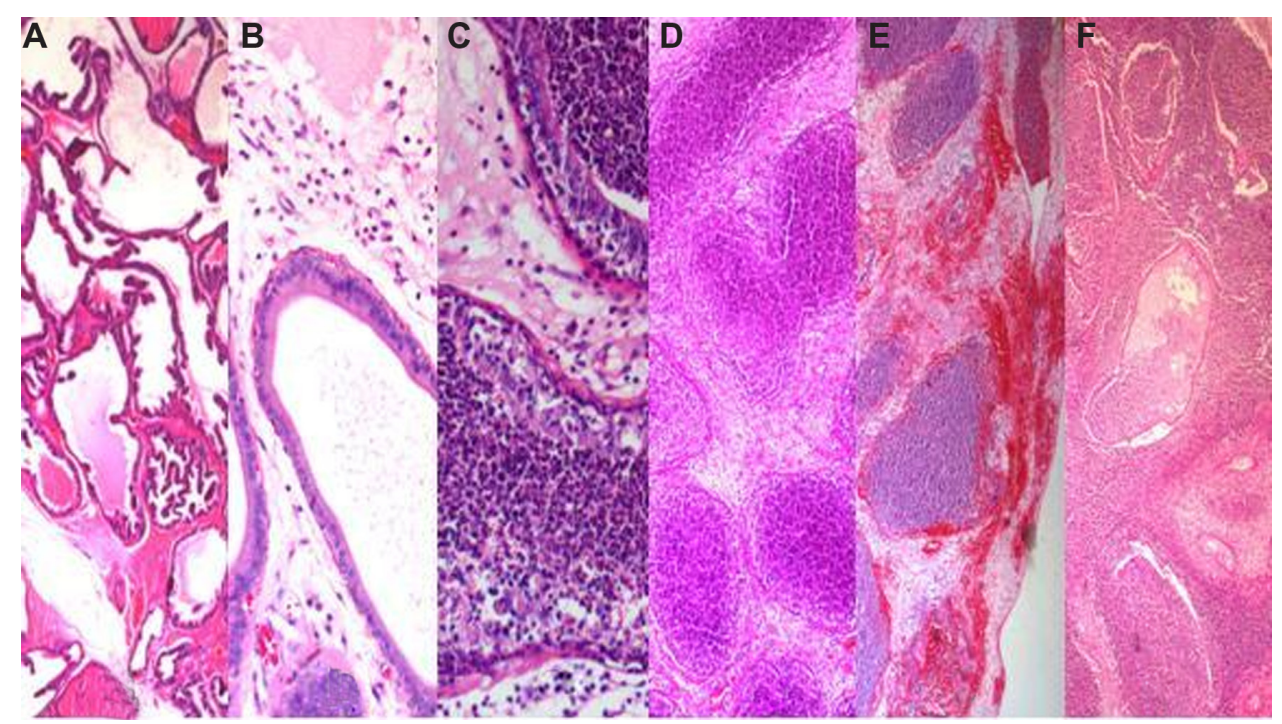

Figure 2 Representative images of different histopathological grades in the rat prostate tissue following UTI.

Notes: Each panel represents a different pathology scored according to the grading scale summarized in Table I. (A) Grade 0 inflammation (H\&E, $\times 4$ ); (B) Grade I inflammation (H\&E, $\times 20)$; (C) Grade 2 inflammation (H\&E, $\times 20)$; (D) Grade 3 inflammation (H\&E, $\times 4)$; (E) Grade 4 inflammation (H\&E, $\times 10) ;(F)$ Grade 5 inflammation (H\&E, $\times 4)$.

Abbreviations: H\&E, hematoxylin and eosin; UTI, urinary tract infection.

prostate from UTI, although not as effectively as the oral administration group.

Here, due to its pharmacokinetic efficacy through oral administration, we chose to study FOF by testing transurethral delivery. The fact that it is directly extracted through the urine without being metabolized in the body strongly suggested that transurethral administration of FOF would be more beneficial than the oral application to a certain extent. ${ }^{17}$ A number of studies have reported on the use of fosfomycin for prophylactic purposes in patients before surgical interventions. ${ }^{18-23}$ Among these, the ones employing prophylactic oral FOF administration reported infection prevention success as $88.6 \%$ to $100 \%$ following transurethral resection of the prostate and urological interventions for diagnostic or treatment purposes. ${ }^{20}$ In another study by Ishizaka et $\mathrm{al}^{18}$ comparing parenteral fosfomycin and

Table 3 Histopathological grades of bladder inflammation in different groups

\begin{tabular}{|c|c|c|c|c|}
\hline \multirow[t]{2}{*}{ Grade } & \multicolumn{4}{|l|}{ Group } \\
\hline & $\begin{array}{l}\text { I } \\
\text { n (\%) }\end{array}$ & $\begin{array}{l}2 \\
\text { n (\%) }\end{array}$ & $\begin{array}{l}\text { Sham } \\
\text { n (\%) }\end{array}$ & $\begin{array}{l}\text { Control } \\
\text { n (\%) }\end{array}$ \\
\hline 0 & $3(50 \%)$ & $4(66.7 \%)$ & $4(66.7 \%)$ & $0(0 \%)$ \\
\hline I & $2(33.3 \%)$ & $2(33.3 \%)$ & $2(33.3 \%)$ & I (16.7\%) \\
\hline 2 & I (I6.7\%) & $0(0 \%)$ & $0(0 \%)$ & I (I6.7\%) \\
\hline 3 & $0(0 \%)$ & $0(0 \%)$ & $0(0 \%)$ & $2(33.3 \%)$ \\
\hline 4 & $0(0 \%)$ & $0(0 \%)$ & $0(0 \%)$ & $2(33.3 \%)$ \\
\hline Total & $6(100 \%)$ & $6(100 \%)$ & $6(100 \%)$ & $6(100 \%)$ \\
\hline
\end{tabular}

Note: The values in bold highlight the most observed histopathological grades. cefotiam, postoperative infection prevention success was reported as $92.9 \%$ in transurethral resection cases, $100 \%$ in open clean surgeries, $84.6 \%$ in open clean-contaminated surgeries, and $33 \%$ in open contaminated surgeries. In studies that used oral FOF as a prophylaxis, FOF was utilized 3 hours before as in our study. In our animal model study, taking the infection intensities in urine and bladder tissue cultures into account, infection prevention success was established as $66 \%$, and considering infection intensity in prostate tissue culture, success was found as $50 \%$ in the oral FOF administration group. To our knowledge, literature from animal studies do not exist on oral FOF treatment following UTI; therefore, in regards to the results from studies conducted on patients and the outcome of the control group one can appreciate that oral FOF use is generally a success in our study. On the other hand, the comparison of the transurethral FOF delivery

Table 4 Histopathological grades of prostate inflammation in different groups

\begin{tabular}{lllll}
\hline Grade & Group & & & \\
\cline { 2 - 5 } & $\mathbf{I}$ & $\mathbf{2}$ & $\begin{array}{l}\text { Sham } \\
\mathbf{n}(\%)\end{array}$ & $\begin{array}{l}\text { Control } \\
\mathbf{n}(\%)\end{array}$ \\
\hline 0 & $\mathbf{n ~ ( \% )}$ & $\mathbf{n ~ ( \% )}$ & $\mathbf{6 ( 1 0 0 \% )}$ & $0(0 \%)$ \\
$\mathrm{I}$ & $\mathrm{I}(16.7 \%)$ & $\mathbf{4 ( 6 6 . 7 \% )}$ & $0(0 \%)$ & $0(0 \%)$ \\
2 & $\mathrm{I}(16.7 \%)$ & $\mathrm{I}(16.7 \%)$ & $0(0 \%)$ & $0(0 \%)$ \\
3 & $\mathrm{I}(16.7 \%)$ & $0(0 \%)$ & $0(0 \%)$ & $\mathrm{I}(16.7 \%)$ \\
4 & $\mathbf{3 ( 5 0 \% )}$ & $\mathrm{I}(16.7 \%)$ & $0(0 \%)$ & $2(33.3 \%)$ \\
5 & $0(0 \%)$ & $0(0 \%)$ & $0(0 \%)$ & $\mathbf{3 ( 5 0 \% )}$ \\
Total & $0(0 \%)$ & $0(0 \%)$ & $6(100 \%)$ & $6(100 \%)$ \\
\hline
\end{tabular}

Note: The values in bold highlight the most observed histopathological grade. 
is more challenging, as it has not been performed in either humans or in animal models. In one study by Leach et $\mathrm{al}^{24}$ that applied FOF topically, a combination of polymyxin B and FOF was demonstrated to decrease ototoxicity risk compared to the use of polymyxin B by itself.

When we evaluated infection intensities in urine and tissue cultures and in bladder and prostate histopathologies, transurethral administration of FOF was not better than its oral administration as we hypothesized earlier. In our opinion this is due to three major concerns: (1) the availability of FOF in the tissue, (2) the predictive value of the rat model, and (3) surgical concerns.

First, when used at the suggested concentrations, FOF remains above minimum inhibitory concentration values for a longer period of time following a single oral FOF administration. On the other hand, the concentration of the drug in urine decreases rapidly with the first urination following transurethral FOF administration. Therefore, direct intravesical injection of a drug that was designed for oral administration might have decreased the efficiency of the drug as some metabolic steps might have been skipped (although sources claim the effectiveness of the drug is unchanged when excreted with urine). ${ }^{17}$

Secondly, for the first time, we established the preventative role of an antibiotic used transurethrally against infections occurring following a transurethral surgical or diagnostic intervention on a rat model of cystitis and prostatitis. Although, the findings show promising results for further use of the model for testing alternative treatment strategies in UTI, one should take the limitations of the model system into consideration such as the rate of spontaneous resolution and other factors related to any other animal model system.

Finally, limitations related to the use of a small animal exist especially regarding the treatment, surgical procedure, and the size of the materials used for surgery. In this study, although a direct replication of transurethral intervention in patients was attempted, factors as the diameter and size of the utilized materials might have induced a higher histological damage than what would have been caused in human patients. Therefore, taking these factors into account, we suggest that transurethral administration would be beneficial and the efficiency could be further increased by using a similar administration strategy by keeping the active agent in the bladder longer or by using a compound designed for transurethral use.

In conclusion, we demonstrated in a rat model of UTI that transurethral administration of an antimicrobial agent prior to the intervention of the bladder and urethra is effective and provides a promising alternative to oral treatment for certain risk conditions. Although, we were not able to show any benefit of using transurethral delivery of FOF over oral treatment, given the risk factors, an improved transurethral delivery using different active agents or by developing strategies to prolong the activity of the same agent in the bladder should be considered. As urine concentration of the active compound is reached more rapidly using transurethral delivery compared to oral treatment, one can consider using a ready-to-use form, such as the lidocaine gels administered with an applicator facilitating the workload of the clinician in high-capacity clinics.

\section{Acknowledgments/disclosures}

We are indebted to Prof Dr James R Johnson for his permission to use the uropathogenic E. coli J96 strain. The authors report no conflicts of interest in this work.

\section{References}

1. Bootsma AM, Laguna Pes MP, Geerlings SE, Goossens A. Antibiotic prophylaxis in urologic procedures: a systematic review. Eur Urol. 2008;54(6):1270-1286.

2. Grabe M. Controversies in antibiotic prophylaxis in urology. Int $J$ Antimicrob Agents. 2004;23 Suppl 1:S17-S23.

3. Almallah YZ, Rennie CD, Stone J, Lancashire MJ. Urinary tract infection and patient satisfaction after flexible cystoscopy and urodynamic evaluation. Urology. 2000;56(1):37-39.

4. Clark KR, Higgs MJ. Urinary infection following out-patient flexible cystoscopy. Br J Urol. 1990;66(5):503-505.

5. Naber KG, Hofstetter AG, Brühl P, et al; for Working group 'Urinary Tract Infections' of the Paul Erlich Societ for Chemotherapy; for Working group 'Urological Infections' of the German Society for Urology. Guidelines for the perioperative prophylaxis in urological interventions of the urinary and male genital tract. Int J Antimicrob Agents. 2001;17(4):321-326.

6. Patel SS, Balfour JA, Bryson HM. Fosfomycin tromethamine. A review of its antibacterial activity, pharmacokinetic properties and therapeutic efficacy as a single-dose oral treatment for acute uncomplicated lower urinary tract infections. Drugs. 1997;53(4):637-656.

7. Dubini F, Riviera L. Treatment of experimental cystitis in the rat with a single dose of fosfomycin trometamol. Chemioterapia. 1988; 7(1):24-28.

8. Arda M. Temel Mikrobiyoloji. 2nd ed. Ankara, Turkey: Medisan Press; 2000.

9. Babacan O. Detection of virulence factors of Escherichia coli strains isolated from urogenital system infections in dogs and cats. Doctoral Thesis. Ankara University Health Science Institute. Ankara, Turkey, 2011.

10. Yamamoto S, Terai A, Yuri K, Kurazono H, Takeda Y, Yoshida O. Detection of urovirulence factors in Escherichia coli by multiplex polymerase chain reaction. FEMS Immunol Med Microbiol. 1995;12(2): 85-90.

11. Johnson JR, Stell AL. Extended virulence genotypes of Escherichia coli strains from patients with urosepsis in relation to phylogeny and host compromise. J Infect Dis. 2000;181(1):261-272.

12. Bauer RJ, Zhang L, Foxman B, et al. Molecular epidemiology of 3 putative virulence genes for Escherichia coli urinary tract infection-usp, iha, and iroN (E. coli). J Infect Dis. 2002;185(10):1521-1524.

13. Hopkins WJ, Gendron-Fitzpatrick A, Balish E, Uehling DT. Time course and host responses to Escherichia coli urinary tract infection in genetically distinct mouse strains. Infect Immun. 1998;66(6): 2798-2802. 
14. Nelson LK, D’Amours GH, Sproule-Willoughby KM, Morck DW, Ceri H. Pseudomonas aeruginosa las and rhl quorum-sensing systems are important for infection and inflammation in a rat prostatitis model. Microbiology. 2009;155(Pt 8):2612-2619.

15. Afşar I, Gönül B, Şener AG, Türker M. In-vitro susceptibility of clinical isolates of escherichia coli to fosfomycin trometamol and other antibiotics. ANKEM Derg. 2005;19(2):77-79.

16. Knottnerus BJ, Nys S, Ter Riet G, Donker G, Geerlings SE, Stobberingh E. Fosfomycin tromethamine as second agent for the treatment of acute, uncomplicated urinary tract infections in adult female patients in The Netherlands? J Antimicrob Chemother. 2008;62(2):356-359.

17. Bergan T, Thorsteinsson SB, Albini E. Pharmacokinetic profile of fosfomycin trometamol. Chemotherapy. 1993;39(5):297-301.

18. Ishizaka K, Kobayashi S, Machida T, Yoshida K. Randomized prospective comparison of fosfomycin and cefotiam for prevention of postoperative infection following urological surgery. J Infect Chemother. 2007;13(5):324-331.

19. Shinagawa N, Mizuno I, Fukui T, et al. Prophylactic effect of fosfomycin on postoperative infection in gastroenterological surgery. Jpn J Antibiot. 2006;59(6):417-427.
20. Selvaggi FP, Battaglia M, Grossi FS, Disabato G, Cormio L. Oral prophylaxis with fosfomycin trometamol in transurethral prostatectomy and urological maneuvers: literature review and personal experience. Infection. 1992;20 Suppl 4:S321-S324.

21. Baert L, Billiet I, Vandepitte J. Prophylactic chemotherapy with fosfomycin trometamol versus placebo during transurethral prostatic resection. Infection. 1990;18 Suppl 2:S103-S106.

22. Periti P, Novelli A, Reali EF, Lamanna S, Fontana P. Prophylactic chemotherapy with fosfomycin trometamol salt during transurethral prostatic surgery: a controlled multicenter clinical trial. Eur Urol. 1987; 13 Suppl 1:S122-S131.

23. di Silverio F, Ferrone G, Carati L. Prophylactic chemotherapy with fosfomycin trometamol during transurethral surgery and urological manoeuvres. Results of a multicentre study. Infection. 1990;18 Supp1 2: S98-S102.

24. Leach JL, Wright CG, Edwards LB, Meyerhoff WL. Effect of topical fosfomycin on polymyxin B ototoxicity. Arch Otolaryngol Head Neck Surg. 1990;116(1):49-53.
Drug Design, Development and Therapy

\section{Publish your work in this journal}

Drug Design, Development and Therapy is an international, peerreviewed open-access journal that spans the spectrum of drug design and development through to clinical applications. Clinical outcomes, patient safety, and programs for the development and effective, safe, and sustained use of medicines are a feature of the journal, which

\section{Dovepress}

has also been accepted for indexing on PubMed Central. The manuscript management system is completely online and includes a very quick and fair peer-review system, which is all easy to use. Visit http://www.dovepress.com/testimonials.php to read real quotes from published authors.

Submit your manuscript here: http://www.dovepress.com/drug-design-development-and-therapy-journal 